\title{
IMPACTO DO TIPO DE EDULCORANTE SOBRE A ACEITAÇÃO DE BISCOITOS DIETÉTICOS JUNTO A CONSUMIDORES PORTADORES E NÃO PORTADORES DE DIABETES MELLITUS
}

\author{
FERNANDA CARVALHO DE SANTANA* \\ JANAÍNA VALÉRIA DA SILVA** \\ VANESSA CIBELLE BARBOZA DE CARVALHO*** \\ MAYARA LÚCIA DA COSTA LEITE MARINS $S^{* * * *}$ \\ ELMA REGINA DA SILVA ANDRADE WARTHA***** \\ PAULO SÉRGIO MARCELLINI $I^{* \star * * * \star}$ \\ MARIA APARECIDA AZEVEDO PEREIRA DA SILVA ${ }^{\star \star \star * \star * *}$
}

\begin{abstract}
Avaliou-se a aceitação quanto a parâmetros sensoriais de formulações de biscoitos dietéticos com diferentes edulcorantes e adição de polidextrose por consumidores portadores e não portadores de Diabetes Mellitus. Os resultados indicaram que o uso da polidextrose como agente de corpo aumentou significativamente a aceitação dos biscoitos $(p \leq 0,05)$ pelos indivíduos que não consomem com frequência produtos dietéticos. Os biscoitos dietéticos avaliados por consumidores portadores de Diabetes apresentaram níveis de aceitação significativamente superiores $(p \leq 0,05)$ aos não portadores da doença para a maioria dos atributos analisados. Os dados de mapa interno de preferencia gerados na análise sensorial sugeriram maior tendência dos consumidores habituais de edulcorantes a apresentarem julgamento diferenciado.
\end{abstract}

PALAVRAS-CHAVE: ANÁLISE SENSORIAL; BISCOITO DIETÉTICO; EDULCORANTE.

* Nutricionista, Mestre em Ciência e Tecnologia de Alimentos, Doutoranda em Ciência dos Alimentos, Faculdade de Ciências Farmacêuticas, Universidade de São Paulo, São Paulo, SP (e-mail: f.santana@ usp.br).

** Nutricionista, Mestre em Ciência e Tecnologia de Alimentos, Departamento de Tecnologia de Alimentos (DTA), Universidade Federal de Sergipe (UFS), São Cristovão, SE (e-mail: javalsi@gmail.com).

*** Nutricionista, Mestranda em Ciências Fisiológicas, Departamento de Fisiologia, UFS, São Cristovão, SE (e-mail: cibelle.b@hotmail.com).

**** Nutricionista, Mestranda em Ciência e Tecnologia de Alimentos, DTA, São Cristovão, SE (e-mail: mayaranutricao@yahoo.com.br).

***** Professora, Doutora em Ciência dos Alimentos, Núcleo de Nutrição, UFS, São Cristovão, SE (e-mail: ewartha@ufs.br).

******Professor, Doutor em Alimentos e Nutrição, Universidade Federal do Estado do Rio de Janeiro, Rio de Janeiro, RJ (e-mail: marcellinips@yahoo.com).

******* Professora, Doutora em Ciência e Tecnologia de Alimentos, DTA, São Cristovão, SE (e-mail: mariasi@ fea.unicamp.br). 


\section{INTRODUÇÃO}

O consumo de alimentos dietéticos ou dos alimentos com adição reduzida de determinados ingredientes (chamados produtos light) vêm ganhando mercado junto a consumidores com restrições dietéticas e aqueles que buscam a manutenção ou mudança na forma física.

Segundo a Portaria n. 29, de 13 de janeiro de 1998, os alimentos dietéticos no Brasil são qualificados como alimentos para fins especiais. São especialmente processados e suas formulações alteradas de forma a se adequarem à utilização em dietas diferenciadas que atendam às necessidades de pessoas em condições metabólicas e fisiológicas específicas (BRASIL, 1998a). Em formulações destinadas a atender indivíduos com distúrbio no metabolismo de açúcares, o teor máximo de açúcar permitido é de $0,5 \mathrm{~g} / 100 \mathrm{~g}$ ou $100 \mathrm{~mL}$ do produto final a ser consumido, quer se trate de sacarose, frutose ou glicose (BRASIL, 1998b). Entretanto, quando se retira a sacarose da formulação de produto alimentícios, normalmente torna-se necessária a adição de dois grupos distintos de compostos, os edulcorantes e os agentes de corpo (VISSOTTO, 2005). A infinidade de adoçantes comerciais disponíveis, com diferentes associações entre edulcorantes, permite a obtenção de características sensoriais e tecnológicas diferenciadas. Assim, a escolha do tipo e da concentração do edulcorante a ser utilizado em alimentos dietéticos assume impacto sobre a aceitação do produto por consumidores.

Os edulcorantes usados pelas indústrias de alimentos devem apresentar propriedades funcionais e sensoriais semelhantes às da sacarose (como gosto doce, baixa densidade calórica, ser fisiologicamente inerte para contribuir no controle da diabetes; ou prevenir cárie dentária) e serem comercialmente viáveis (MALIK, JEYARANI e RAGHAVAN, 2002). Devem, ainda, apresentar estabilidade em diferentes condições de $\mathrm{pH}$ e temperatura, além de interagirem com amidos e proteínas de forma similar aos açúcares (CAMPOS, 2000).

Embora a utilização do edulcorante muitas vezes não promova alterações significativas no sabor do alimento formulado, provoca mudanças perceptíveis nas características de textura e aparência do produto (OLIVEIRA et al., 2004). Assim, notadamente na formulação de produtos dietéticos ou light destinados à panificação (como bolos e biscoitos), além dos edulcorantes torna-se necessária a adição de ingredientes com propriedades de agente de corpo para minimizar problemas com o volume e a textura do produto final (BATTOCHIO et al. 2006). A polidextrose constitui um dos agentes de corpo mais utilizados pela indústria de alimentos moderna.

As propriedades sensoriais são influenciadas diretamente pela composição química e propriedades físicas dos alimentos, que determinam a competitividade de produtos alimentícios no mercado consumidor. Assim, testes sensoriais são usualmente empregados para se avaliar a qualidade e aceitação dos alimentos. Por meio de testes sensoriais pode-se identificar a presença ou ausência de defeitos (que prejudiquem a aceitação do produto junto ao consumidor), ou detectar particularidades que não podem ser verificadas por outros procedimentos analíticos (MUÑOZ, CIVILLE e CARR, 1992).

Os testes sensoriais afetivos avaliam basicamente a aceitação ou a preferência dos consumidores por um ou mais produtos. Esses testes têm a finalidade de determinar o quanto 0 consumidor gostou ou desgostou de determinado produto, qual o produto mais apreciado e quais as características sensoriais que determinam a preferência de certo público-alvo, consumidores habituais ou potenciais do produto analisado (STONE \& SIDEL, 2004; MEILGAARD, CIVILLE e CARR, 1999).

O objetivo deste estudo foi investigar o efeito da substituição do açúcar por adoçantes comercias, com ou sem uso de polidextrose, sobre a aceitação de biscoitos dietéticos junto a dois grupos de consumidores: portadores e não portadores de Diabetes Mellitus. 


\section{MATERIAL E MÉTODOS}

\subsection{AMOSTRAS}

Os biscoitos foram elaborados seguindo-se formulação desenvolvida em estudo prévio de otimização dos ingredientes sacarose, fécula de mandioca, farinha de trigo e de casca de maracujá por delineamento fatorial, associado à análise por Metodologia Superfície de Resposta por 51 indivíduos saudáveis (SANTANA et al., 2011). A formulação otimizada continha: sacarose (115 g), farinha de trigo (145 g), fécula de mandioca $(39,06 \mathrm{~g})$, farinha da casca de maracujá $(39,06 \mathrm{~g})$, fermento químico $(5 \mathrm{~g})$, margarina $(67,5 \mathrm{~g})$ e sumo de maracujá $(45 \mathrm{~mL})$.

Para a elaboração do biscoito dietético, a formulação otimizada teve a sacarose substituída pelos seguintes edulcorantes comerciais e termoestáveis: i) formulação comercial constituída de sacarina sódica e ciclamato de sódio (SCS), maltodextrina e regulador de acidez citrato de sódio, ii) formulação comercial contendo sucralose e acesulfame K (SUC), e iii) formulação comercial à base de esteviosídeos (STE), contendo também maltodextrina e anti-umectante dióxido de silício. A quantidade de cada edulcorante foi estimada com base nos valores de equivalência de doçura especificados pelo fabricante, em que $100 \mathrm{~g}$ de sacarose equivale a $40 \mathrm{~g}$ do edulcorante STE, $45 \mathrm{~g}$ de SUC e 13,34 g de SCS.

Foram elaboradas seis diferentes formulações de biscoito dietético, especificadas na Tabela 1.

O aditivo alimentar polidextrose foi utilizado com o objetivo de restituir à formulação original a quantidade total de açúcar que foi substituído com a utilização do edulcorante. Neste estudo, foi utilizado polidextrose da marca Litesse ${ }^{\mathrm{TM}}$, gentilmente cedida pela empresa Danisco Brasil (São Paulo, SP).

\section{TABELA 1 - INGREDIENTES E RESPECTIVAS QUANTIDADES UTILIZADAS NO DESENVOLVIMENTO DAS FORMULAÇÕES DO BISCOITO DIETÉTICO}

\begin{tabular}{lcccccc}
\hline \multirow{2}{*}{ Ingredientes } & \multicolumn{7}{c}{ Formulação $^{1}$} \\
& BSUC & BSUCP & BSCS & BSCSP & BSTE & BSTEP \\
\hline Adoçante & & & & & & \\
$\quad$ Tipo & SUC & SUC & SCS & SCS & STE & STE \\
$\quad$ Quantidade (g) & 14,44 & 14,44 & 15,33 & 15,33 & 46 & 46 \\
Polidextrose (g) & - & 101 & - & 99,67 & - & 69 \\
Fécula de mandioca (g) & 39,06 & 39,06 & 39,06 & 39,06 & 39,06 & 39,06 \\
Farinha da casca do maracujá (g) & 39,06 & 39,06 & 39,06 & 39,06 & 39,06 & 39,06 \\
Farinha de trigo (g) & 145,08 & 145,08 & 145,08 & 145,08 & 145,08 & 145,08 \\
Fermento químico $(\mathrm{g})$ & 5 & 5 & 5 & 5 & 5 & 5 \\
Sumo de maracujá $(\mathrm{mL})$ & 45 & 45 & 45 & 45 & 45 & 45 \\
Margarina (g) & 67,5 & 67,5 & 67,5 & 67,5 & 67,5 & 67,5 \\
\hline
\end{tabular}

${ }^{1} \mathrm{BSUC}=$ biscoito contendo a mistura de edulcorantes sucralose e acessulfame-K, BSUCP $=$ biscoito contendo a mistura de edulcorantes sacarina e ciclamato de sódio e polidextrose; BSCS = biscoito contendo o edulcorante estévia; BSCSP = biscoito contendo a mistura de edulcorantes sucralose e acessulfame-K e polidextrose, BSTE = biscoito contendo a mistura de edulcorantes sacarina e ciclamato de sódio; e BSTEP = biscoito contendo o edulcorante estévia e polidextrose. ${ }^{2} \mathrm{SUC}=$ Adoçante contendo a mistura de edulcorantes sucralose e acessulfame-K; STE = Adoçante contendo o edulcorante estévia e, SCS = Adoçante contendo a mistura de edulcorantes ciclamato e sacarina sódica.

\subsection{TESTE AFETIVO}

Foram recrutados 117 consumidores potenciais de biscoitos, sendo 97 não portadores e 20 portadores de Diabetes Mellitus tipo 1 e/ou 2 (DM). Participaram da análise sensorial os consumidores que assinaram o Termo de Consentimento Livre e Esclarecido, aprovado pelo Comitê 
de Ética da Universidade Federal de Sergipe (São Cristovão, SE), n CAAE 0117.0.107.000-10, conforme a Resolução CNS n¹96/96. Adotou-se como critério de inclusão: idade entre 18 e 60 anos, interesse e disponibilidade de tempo, gostar de biscoito e de maracujá. Antes da realização do teste sensorial, os voluntários responderam questionário para avaliação do consumo alimentar com foco no consumo de edulcorantes e produtos dietéticos.

As amostras foram avaliadas quanto a aceitação dos parâmetros aparência, aroma, sabor, textura e impressão global utilizando-se escala hedônica estruturada de nove pontos ( 9 = gostei muitíssimo; 5 = nem gostei/nem desgostei; 1 = desgostei muitíssimo) e escala de intenção de compra de sete pontos $(7=$ certamente compraria, $4=$ talvez comprasse/talvez não comprasse, 1 = certamente não compraria) (STONE \& SIDEL, 2004; MEILGAARD, CIVILLE e CARR, 1999).

Todos os consumidores (117) avaliaram as seis formulações de forma monádica, em pratos plásticos descartáveis, codificados com números de três algarismos, havendo sempre um copo de água potável e pedaços de pão branco disponíveis para enxágue bucal. Adotou-se a ordem de apresentação das formulações balanceada para os efeitos de primeira ordem, seguindo-se delineamento de MacFie et al. (1989).

Para os consumidores não portadores de DM, o teste de aceitação foi realizado em cabines individuais do Laboratório de Técnica Dietética da Universidade Federal de Sergipe (UFS) e para os portadores de diabetes adaptou-se o local para que os participantes estivessem distantes um do outro e obtivessem conforto térmico e visual, já que não havia cabines individuais nas dependências dos ambulatórios de nutrição e endocrinologia do Hospital Universitário da UFS (Aracaju, SE).

\subsection{ANÁLISE ESTATÍSTICA}

Os resultados do teste de aceitação foram avaliados por análise estatística univariada (ANOVA - análise de variância) e depois por Testes de médias de Tukey ao nível de confiança de $95 \%(p \leq 0,05)$. Análise estatística multivariada, utilizando a técnica do Mapa Interno de Preferência (GREENHOFF e MACFIE, 1994) foi realizada para os dados de aceitação dos atributos sabor, textura, aceitação global e intenção de compra com auxílio dos programas estatísticos SAS (2009) e STATISTICA 9.0 (STATSOFT, 2009).

\section{RESULTADOS E DISCUSSÃO}

\subsection{PERIL DEMOGRÁFICO E COMPORTAMENTAL DOS PARTICIPANTES QUANTO AO CONSUMO}

Caracterizar os grupos de consumidores de acordo com variáveis sócio-demográficas, de saúde e de consumo de alimentos dietéticos assume grande importância para o conhecimento do mercado potencial dos produtos formulados. Assim, após segmentação dos grupos de indivíduos com (PDM) e sem (NPD) a doença verificou-se que a maioria de consumidores pertencia ao gênero feminino. Entretanto, enquanto cerca de $90 \%$ dos indivíduos do grupo PDM encontrava-se na faixa etária entre 50 e 60 anos, sendo casados (60\%), o grupo NPD foi formado por indivíduos na faixa etária entre 20 e 30 anos (62\%) e solteiros (85\%).

Entre os consumidores portadores de diabetes, $70 \%$ declararam diagnóstico clínico de diabetes tipo 2, 85\% desses obtiveram diagnóstico clínico há mais de 5 anos e utilizavam algum medicamento hipoglicemiante à época da pesquisa. Esses resultados são similares aos reportados por Souza (2006), pois $87,7 \%$ dos pacientes participantes de seu estudo declararam ter diabetes tipo 2 e $7,7 \%$ diabetes tipo 1. De fato, o diabetes tipo 2 prevalece entre pessoas com maior idade, como comprovaram Castro e Franco (2002) e Souza (2006).

Quanto aos hábitos de consumo, Tabela 2, comprovou-se que $100 \%$ dos diabéticos consumiam edulcorantes, enquanto apenas $24 \%$ dos não portadores de diabetes consumiam 
edulcorantes. Entre os diabéticos, 90\% preferiam edulcorantes à base de aspartame e 10\% edulcorantes à base de esteviosídeos. Todos os indivíduos (PDM e NPD) quando utilizavam edulcorantes, o faziam para adoçar bebidas como suco, café e vitamina, dentre outros. Entre os diabéticos, 20\% ainda utilizavam edulcorantes para o preparo de sobremesas que não iam ao fogo e $10 \%$ em alimentos submetidos à cocção.

Em relação aos alimentos dietéticos, 65\% dos PDM e 22\% dos NPD consumiam esse tipo de produto. A proporção de consumidores diabéticos (35\%) que não utilizavam produtos dietéticos foi superior à encontrada por Castro e Franco (2002) e por Souza (2006), respectivamente, 24,2\% e 23,1\%.

Dentre os alimentos dietéticos mais consumidos encontram-se os refrigerantes, doces e iogurte. Resultados similares foram obtidos por loshii e Toledo (1994), Souza (2006) e Oliveira e Franco (2010) que verificaram maior consumo de refrigerante diet, seguido por gelatina diet entre pacientes diabéticos.

Em seus estudos com portadores de diabetes melittus, Castro e Franco (2002) constataram que $90,5 \%$ deles adoçavam suas bebidas com edulcorantes. A combinação sacarina e ciclamato de sódio era usada por $45,2 \%$ dos pacientes, enquanto $29,3 \%$ consumiam aspartame. Entre os 65 diabéticos entrevistados por Souza (2006), a maioria (92,3\%) utilizava edulcorantes para adoçar líquidos e apenas $7,7 \%$ não consumiam esse tipo de produto por considerarem seu sabor ruim. 0 aspartame era preferido por $38,3 \%$ dos usuários de edulcorante, $28,3 \%$ usavam a combinação de sacarina e ciclamato e $11,7 \%$ consumiam esteviosídio.

\section{TABELA 2 - CARACTERIZAÇÃO DO CONSUMO ALIMENTAR DOS PARTICIPANTES DIABÉTICOS E NÃO DIABÉTICOS DOS TESTES AFETIVOS COM OS BISCOITOS DIETÉTICOS}

\begin{tabular}{lccc}
\hline \multicolumn{1}{c}{ Variáveis do consumo alimentar } & $\begin{array}{c}\text { Total \% } \\
(\mathbf{n = 1 1 7 )}\end{array}$ & $\begin{array}{c}\text { Grupo } \\
\text { PDM \% } \\
(\mathbf{n = 2 0})\end{array}$ & $\begin{array}{c}\text { Grupo } \\
\text { NPD \% } \\
\text { (n=97) }\end{array}$ \\
\hline Consumo adoçante & & 100 & 24 \\
Sim & 37 & - & 76 \\
Não & 63 & 90 & 14 \\
Edulcorante & 27 & 10 & 2 \\
Aspartame & 3 & - & 5 \\
Estévia & 4 & - & 2 \\
Ciclamato e sacarina sódica & 2 & 5 & 6 \\
Sucralose e acessulfame-K & & 5 & 3 \\
Frequência & 6 & 10 & 1 \\
< 1dia/semana & 3 & 80 & 13 \\
1-3 dias & 3 & 100 & 100 \\
3-5 dias & 25 & 20 & - \\
Todos os dias & & 10 & - \\
Situação de uso* & 37 & 65 & 22 \\
Líquido & 3 & 35 & 78 \\
Sobremesas não submetidas à cocção & 2 & & \\
Sobremesas submetidas à cocção & & & \\
Consumo de alimentos diet & 29 & & \\
Sim & 71 & & \\
Não & &
\end{tabular}

*mais de uma opção foi declarada.

Ioshii e Toledo (1994), pesquisando o uso de edulcorantes entre consumidores paranaenses verificaram a preferência pela combinação sacarina e ciclamato de sódio, seguida pelo aspartame.

Ao analisarem o consumo de edulcorantes e produtos dietéticos por indivíduos com 
Diabetes Mellitus tipo 2, atendidos pelo Sistema Único de Saúde em Ribeirão Preto (SP), Oliveira e Franco (2010) verificaram que $76,7 \%$ dos pacientes faziam uso de edulcorantes e $50,8 \%$ deles consumiam produtos dietéticos.

\subsection{TESTE DE ACEITAÇÃO: CONSUMIDORES NÃO ESTRATIFICADOS}

Os resultados dos parâmetros sensoriais avaliados estão expressos na Tabela 3. Para a aparência e o aroma não houve diferença significativa $(p \leq 0,05)$ entre as formulações e todas as médias situaram-se ligeiramente acima do valor 6 (gostei ligeiramente) e abaixo do valor 7,0 (gostei moderadamente) da escala hedônica.

O atributo textura foi o menos apreciado, notadamente nas formulações BSUC e BSTE, cujas médias variaram entre 4,0 ("desgostei ligeiramente") e 6,33 (valor próximo ao termo correspondente a "gostei ligeiramente") na escala hedônica.

A polidextrose melhorou significativamente $(p \leq 0,05)$ a aceitação da textura das formulações BSUCP e BSCSP, porém não alterou significativamente $(p \leq 0,05)$ a aceitação da textura dos biscoitos formulados com estévia. A polidextrose possibilita a manutenção do teor de sólidos solúveis em alimentos processados nos quais a sacarose foi substituída por edulcorantes, melhorando sua textura.

Em relação à aceitação do atributo sabor, nota-se que as formulações BSCSP e BSUCP foram as mais apreciadas pelos participantes do teste sensorial.

\section{TABELA 3 - VALORES MÉDIOS DE ACEITAÇÃO DOS ATRIBUTOS SENSORIAIS DOS BISCOITOS DIETÉTICOS RICOS EM FIBRAS E FORMULADOS COM DIFERENTES EDULCORANTES ( $\mathrm{N}=117$ CONSUMIDORES)}

\begin{tabular}{|c|c|c|c|c|c|}
\hline \multirow[b]{2}{*}{ Amostras $^{2}$} & \multicolumn{5}{|c|}{ Atributo Sensorial $^{1}$} \\
\hline & Aparência & Aroma & Sabor & Textura & $\begin{array}{c}\text { Impressão } \\
\text { global }\end{array}$ \\
\hline BSUC & 6,34 ab & $6,18 \mathrm{ab}$ & $5,37^{b}$ & $4,16^{\mathrm{c}}$ & $5,45^{c}$ \\
\hline BSUCP & 6,79 a & $6,68^{a}$ & $6,57^{a}$ & $5,63 a b$ & $6,60 \mathrm{ab}$ \\
\hline BSCS & $6,58 \mathrm{ab}$ & $6,29 a b$ & $4,82^{b}$ & $6,33^{a}$ & $5,70^{c}$ \\
\hline BSCSP & 6,89 a & $6,74^{\mathrm{a}}$ & $6,70^{a}$ & $5,48^{\mathrm{b}}$ & $6,75^{a}$ \\
\hline BSTE & $6,11^{\mathrm{b}}$ & $5,95^{b}$ & $5,20^{b}$ & $4,42^{c}$ & $5,58^{c}$ \\
\hline BSTEP & $6,82^{a}$ & $6,53^{a b}$ & $5,33^{\mathrm{b}}$ & 5,66 ab & $6,00^{\mathrm{bc}}$ \\
\hline $\mathrm{DMS}^{3}$ & 0,6006 & 0,6281 & 0,7531 & 0,8463 & 0,6986 \\
\hline
\end{tabular}

${ }^{1}$ Médias com letras em comum na mesma coluna não diferem entre si a $p \leq 0,05$ pelo teste de Tukey, ${ }^{2} \mathrm{BSUC}=$ biscoito contendo a mistura de edulcorantes sucralose e acessulfame-K, BSUCP = biscoito contendo a mistura de edulcorantes sacarina e ciclamato de sódio e polidextrose; BSCS = biscoito contendo o edulcorante estévia; BSCSP = biscoito contendo a mistura de edulcorantes sucralose e acessulfame-K e polidextrose, BSTE = biscoito contendo a mistura de edulcorantes sacarina e ciclamato de sódio; e BSTEP = biscoito contendo o edulcorante estévia e polidextrose, ${ }^{3} \mathrm{DMS}$ = diferença mínima significativa.

Embora derivados da sacarose, o perfil tempo intensidade da sucralose e do acessulfame caracteriza-se por percepção de doçura rápida ao se colocar o alimento na boca, que persiste por período ligeiramente maior ao da sacarose, porém sem revelar sabor residual amargo ou metálico (CÂNDIDO e CAMPOS, 1996). Já a sacarina apresenta desagradável gosto residual, minimizado pela combinação com ciclamato (TOZZETO, 2005). Assim, as baixas médias de aceitação das formulações 
BSUC e BSTE podem ser atribuídas às diferenças de seus perfis sensoriais, comparativamente ao da sacarose, adoçante do hábito alimentar dos consumidores que participaram do teste.

O aumento da aceitação do sabor dos biscoitos com a adição de polidextrose pode ser atribuído a dois fatores: à doçura conferida pela própria polidextrose, que também tem poder edulcorante, e sua participação na reação de Maillard, gerando produtos que melhoraram a cor e o sabor dos alimentos termicamente processados (PINHEIRO e PENNA, 2004; GOMES et al., 2007).

O sabor do biscoito formulado apenas com estévia, sem adição de polidextrose, obteve média inferior a 5 na escala hedônica ("desgostei ligeiramente" e "nem gostei / nem desgostei"). Essa ocorrência é comum em produtos contendo altos níveis de adição de estévia, devido ao forte gosto residual amargo (CARDELLO, SILVA e DAMASCO, 1999; SILVA et al., 2001). A adição de polidextrose melhorou ligeiramente a aceitação do sabor dos biscoitos formulados com estévia, mais não o suficiente para alcançar significância estatística $(p>0,05)$.

Quanto à aceitação global, os biscoitos formulados com adição de polidextrose obtiveram níveis maiores de aceitação que os biscoitos sem esse ingrediente. $O$ impacto positivo da polidextrose sobre a aceitação global apresentou significância estatística $(p \leq 0,05)$ para as formulações BSUC e BSTE (Tabela 3). Na aceitação global são avaliados todos os atributos conjuntamente e, possivelmente, a textura e o sabor que sofreram influência da polidextrose foram decisivos para o nível de aceitação global das amostras analisadas.

A formulação BSUCP alcançou melhor aceitação, não diferindo significativamente $(p \leq 0,05)$ da BSCSP. A formulação contendo estévia, mesmo após a adição de polidextrose, obteve aceitação global significativamente inferior $(p \leq 0,05)$ aos demais biscoitos.

\subsection{TESTE DE ACEITAÇÃO: CONSUMIDORES ESTRATIFICADOS QUANTO AO DIAGNÓSTICO DE DIABETES MELLITUS}

A Tabela 4 apresenta as médias de aceitação das seis formulações de biscoitos estratificadas por grupo de consumidores que realizaram o teste: 20 diabéticos e 97 não diabéticos. Diferenças entre os dois grupos foram visualizadas para os atributos sabor, textura e impressão global, cujas médias de aceitação dos consumidores diabéticos foram significativamente superiores $(p \leq 0,05)$.

O sabor dos biscoitos BSCS e BSTE foi rejeitado pelos consumidores não portadores de diabetes (Tabela 4), com médias inferiores a 5,0 na escala hedônica, e alcançaram média de aceitação entre 6,0 (gostei ligeiramente) e 7,0 (gostei moderadamente) entre o grupo de diabéticos. Castro e Franco (2002) estudaram a caracterização do consumo de adoçantes alternativos e produtos dietéticos por indivíduos diabéticos. Dos 201 entrevistados, $176(87,6 \%)$ relataram gostar de produtos contendo edulcorante porque se acostumaram com seu uso. Isso justifica a satisfatória aceitação dos biscoitos dietéticos junto ao grupo de diabéticos e demonstra que a introdução de novo ingrediente no plano alimentar pode fazer com que o individuo se adapte à nova situação de consumo.

No que diz respeito à textura, não houve diferença entre as médias de aceitação atribuídas pelos consumidores do grupo portador de diabetes. Entretanto, no grupo dos consumidores não diabéticos, as formulações BSUC e BSAC obtiveram médias significativamente baixas ("desgostei moderadamente" e "desgostei ligeiramente"). A possível explicação para tal fato consiste na menor importância que os consumidores do grupo dos diabéticos parecem dispensar para esse atributo, pois os biscoitos com polidextrose apresentavam textura diferenciada daqueles sem esse agente de massa.

Para o grupo de consumidores diabéticos, as diferenças percebidas no sabor entre as amostras não foi suficiente para influenciar o julgamento da impressão global, em oposição ao grupo dos indivíduos não portadores de diabetes. Esses resultados demonstram a importância de se testar novos produtos junto a todos os segmentos de potenciais consumidores, pois grande diferença pode ocorrer entre os vários segmentos do mercado consumidor. 


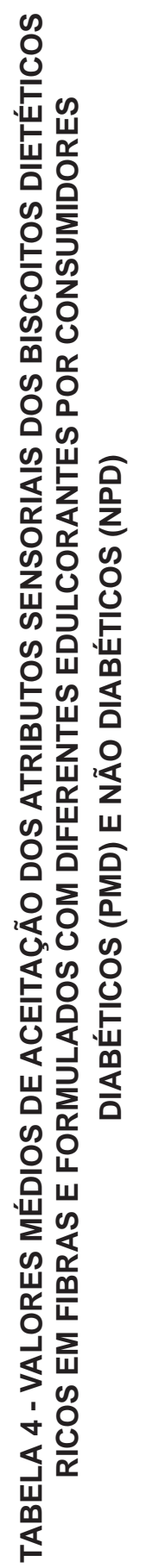

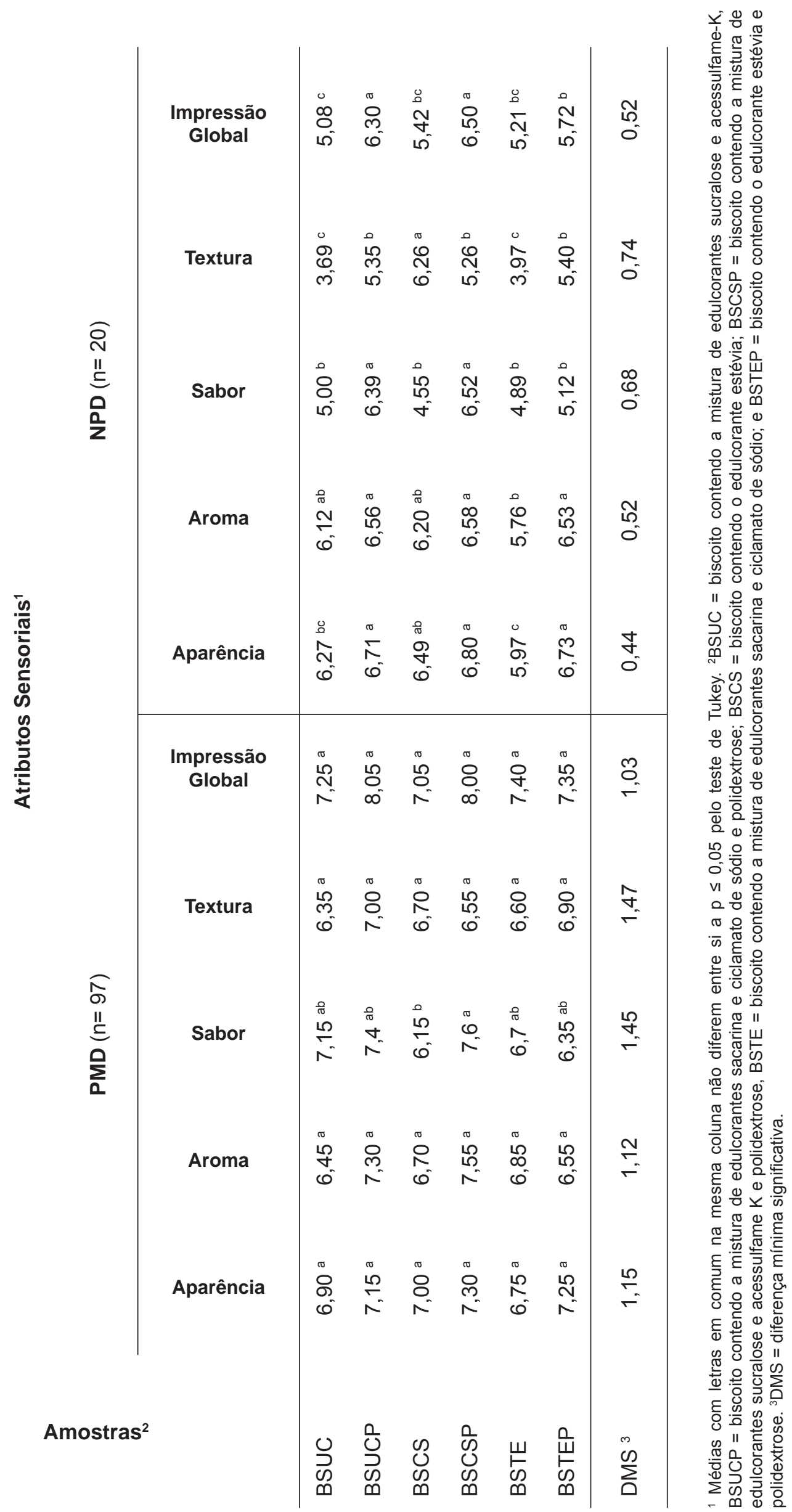


Como segmentos distintos de consumidores apresentam preferências divergentes com relação ao mesmo conjunto de amostras, a simples análise das médias obtidas no teste de aceitação pode deixar de revelar informações importantes. Determinada amostra pode não ter apresentado a maior média de aceitação, mas a preferência de significativo grupo de consumidores demonstra viabilidade mercadológica específica.

Mapas Internos de Preferência (MDPREF) foram gerados para permitir a visualização da preferência de cada consumidor com relação aos atributos sensoriais que mais variaram entre os biscoitos dietéticos formulados (sabor, textura e aceitação global).

As amostras e os consumidores foram alocados em gráficos distintos para melhor visualização dos resultados, mas ambos devem ser interpretados como figuras que se sobrepõe formando apenas um espaço bidimensional de preferência. Na Figura 1 (a), os 97 consumidores não portadores de diabetes estão representados pela letra "N", enquanto os $20 \mathrm{com}$ diagnóstico clínico de Diabete Mellitus tipo 1 ou 2 são representados pela letra "D".
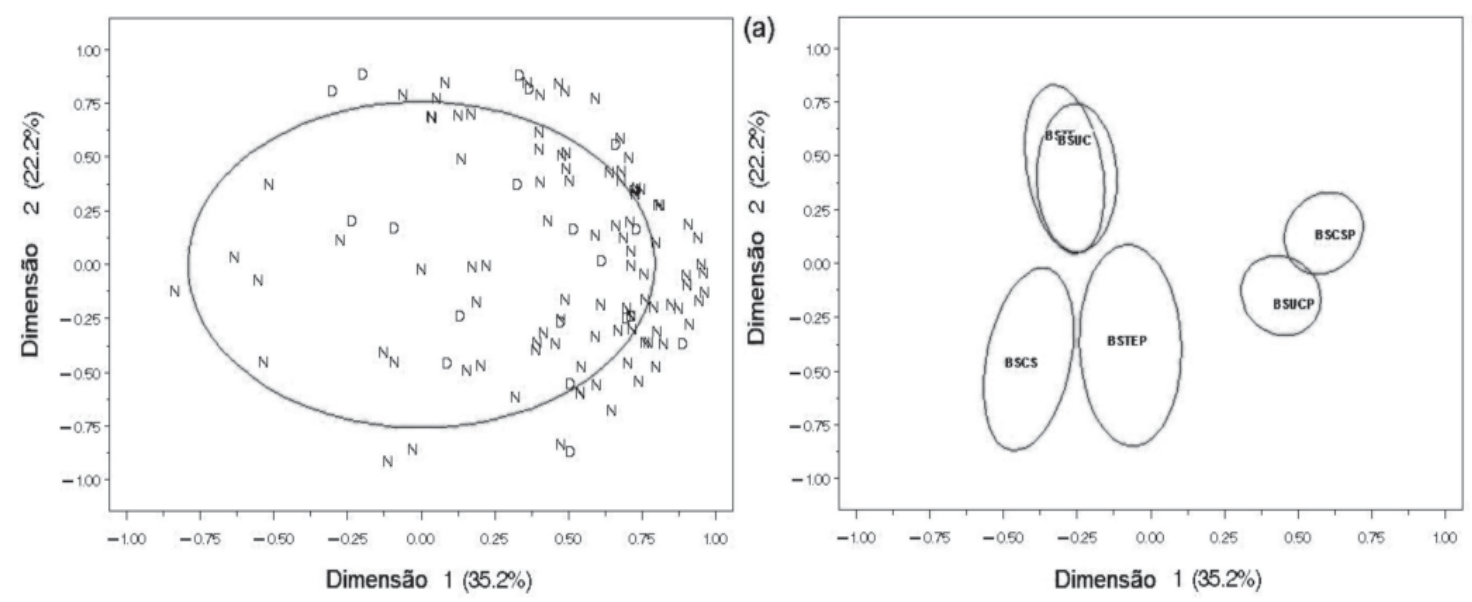

(b)

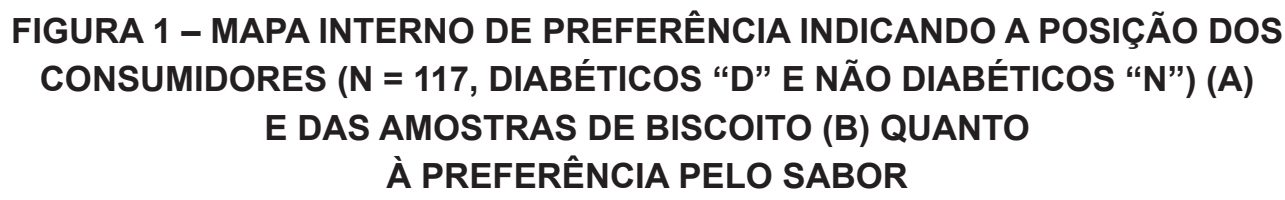

Como o mapa interno de preferência permite a visualização espacial das amostras e consumidores conforme preferência, verificou-se que a maioria dos consumidores localizou-se próximo dos biscoitos BSCSP e BSUCP, indicando que essas amostras foram preferidas pelos consumidores que realizaram o teste. A Tabela 4 já havia apontado que esses dois biscoitos obtiveram maiores médias de aceitação junto aos consumidores. Os demais biscoitos foram preferidos por número marginal de consumidores, sugerindo sua pouca competitividade.

As formulações BSUC e BSTE obtiveram a preferência de proporção irrisória de consumidores, podendo-se inferir que a polidextrose determinou a preferência nesse caso. No entanto, a polidextrose não melhorou a resposta dos consumidores com relação aos biscoitos formulados com estévia pela incapacidade de mascarar o amargor e sabor residual metálico inerentes à estévia.

A elipse visualizada no centro da Figura 1 ( $a$ e b) representa o ajuste dos dados ao modelo utilizado para se obter o MDPREF. Essa elipse estabelece região com $95 \%$ de confiança, estando os consumidores cujas respostas foram significativamente ajustadas $(p \leq 0,05)$ do lado de fora da elipse. A probabilidade desses consumidores terem atribuído valores de aceitação ao acaso aos biscoitos é menor que $5 \%$ (VILLANUEVA, 2003).

A Tabela 5 mostra que dos 117 consumidores, 56 foram significativamente ajustados 
$(p \leq 0,05)$ e concentraram-se nos quadrantes à direita da Figura 1 (superior e inferior) em que estão os biscoitos BSCSP e BSUCP. Não foi possível verificar segmentação entre os indivíduos portadores e não portadores de diabetes que foram ajustados pelo modelo. Esses dois tipos de consumidores localizaram-se próximos dos biscoitos BSCSP e BSUCP, indicando preferência de ambos os grupos.

Dos 56 consumidores ajustados (Tabela 5), 8 pertenciam ao grupo dos diabéticos enquanto 48 não. Isso indica que cerca de $40 \%$ dos 20 consumidores diabéticos que participaram do teste foram ajustados pelo modelo usado para gerar o MDPREF, proporção muito similar aos indivíduos não diabéticos ajustados pelo modelo (aproximadamente $50 \%$ dos 97 voluntários não diabéticos). $A$ despeito dos consumidores diabéticos estarem habituados ao sabor diferenciado dos edulcorantes em relação à sacarose, razão dos maiores níveis de aceitação dos biscoitos que os consumidores normais, verificou-se discriminação similar dos não portadores de diabetes quanto aos biscoitos que mais gostaram e menos gostaram. Mesmo sendo mais tolerantes que os indivíduos não diabéticos ao perfil sensorial diferenciado dos edulcorantes em geral, os diabéticos percebem e valorizam os adoçantes com as melhores características sensoriais.

\section{TABELA 5 - NÚMERO DE CONSUMIDORES SIGNIFICATIVAMENTE AJUSTADOS SITUADOS

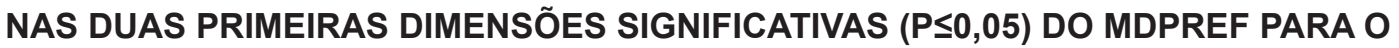 ATRIBUTO SABOR ( $\mathrm{N}=117$ CONSUMIDORES)}

\begin{tabular}{lccccc}
\hline Atributos & $\begin{array}{c}\text { Total } \\
\text { ajustado }\end{array}$ & $\begin{array}{c}\text { Superior } \\
\text { direito }\end{array}$ & $\begin{array}{c}\text { Inferior } \\
\text { direito }\end{array}$ & $\begin{array}{c}\text { Superior } \\
\text { esquerdo }\end{array}$ & $\begin{array}{c}\text { Inferior } \\
\text { esquerdo }\end{array}$ \\
\hline Sabor & 56 & 25 & 25 & 3 & 3 \\
\hline
\end{tabular}

As formulações com maior aceitação e preferência, no que diz respeito ao atributo sabor, foram desenvolvidas com a mistura sucralose e acessulfame-K e polidextrose, bem como as elaboradas com ciclamato e sacarina sódica e polidextrose.

O MDPREF gerado a partir dos dados de aceitação para o atributo textura está apresentado na Figura 2. Verifica-se que as formulações BSUCP, BSCSP e BSTEP apresentam elipses com $95 \%$ de confiança que se sobrepõem, indicando inexistência de diferença estatística $(p>0,05)$ entre os valores de aceitação de textura dessas três formulações. A Tabela 5 já indicava que as médias de aceitação dessas formulações não apresentaram diferença significativa entre si.
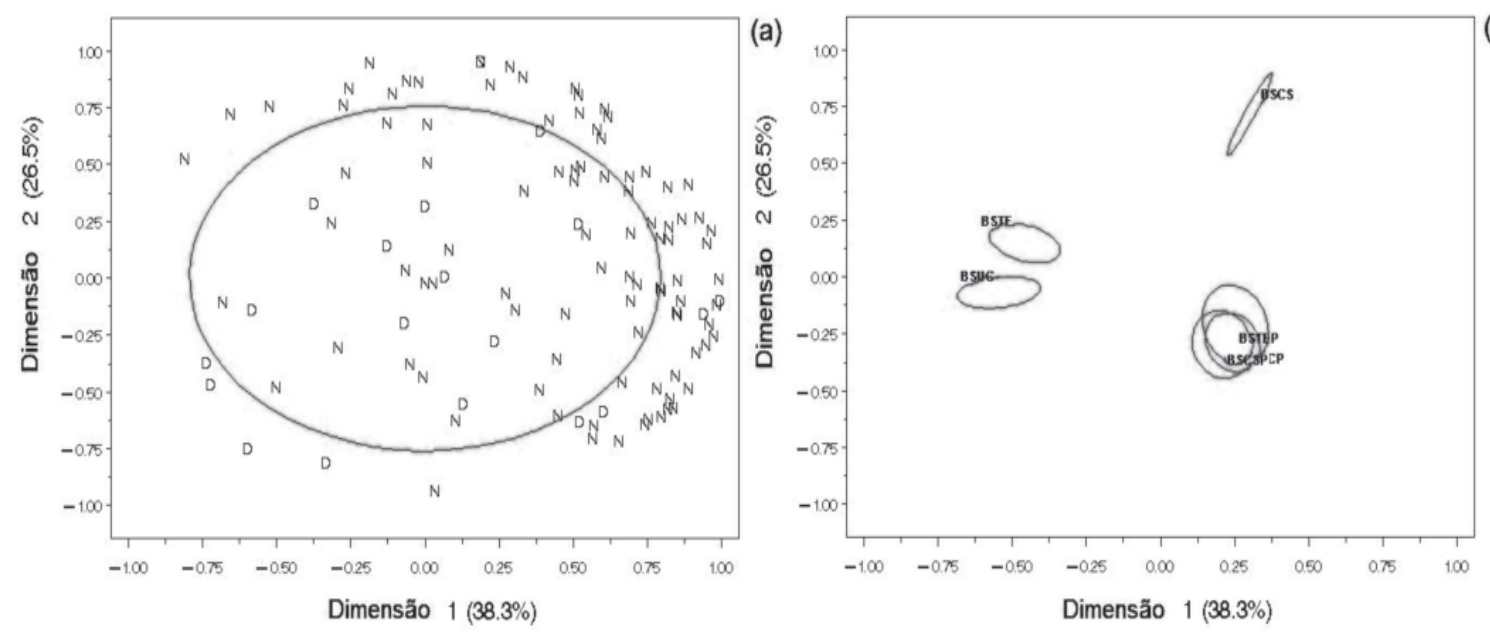

(b)

FIGURA 2 - MAPA INTERNO DE PREFERÊNCIA INDICANDO A POSIÇÃO DOS CONSUMIDORES ( $N=117$, DIABÉTICOS “D” E NÃO DIABÉTICOS “N”) (A) E DAS AMOSTRAS DE BISCOITO (B) QUANTO À PREFERÊNCIA PELA TEXTURA 
As formulações BSUC e BSTE encontram-se no mesmo quadrante e próximas, indicando preferência similar entre os consumidores com relação à textura. Apesar de não ter sido realizada análise instrumental da textura, essas amostras apresentavam aspecto umedecido no interior do biscoito (miolo) e textura mais mole. A formulação BSCS, devido disposição espacial no MDPREF (Figura 2 b), distinguiu-se das demais quanto à textura dos biscoitos.

Os dados dos consumidores analisados por MDPREF indicaram que todos os biscoitos formulados com polidextrose (BSUCP, BSCSP e BSTEP) apresentaram preferência similar ao biscoito formulado apenas com estévia (BSCS), dado que a maioria dos consumidores encontra-se igualmente distribuída entre essas quatro formulações (Figura 3). De fato, a Tabela 6 mostra que dos 72 indivíduos ajustados pelo modelo que gerou o MDPREF, 30 ficaram no quadrante superior direito junto com a formulação BSCS e 28 no quadrante inferior direito no qual se encontram as formulações BSUCP, BSCSP e BSTEP. Isso demonstra a importância de se analisar os dados hedônicos coletados junto aos consumidores por testes adicionais aos de médias, como Tukey e t-student. Esses resultados evidenciam também a ação benéfica da polidextrose sobre a textura dos alimentos elaborados com a substituição da sacarose por edulcorantes.

\section{TABELA 6 - NÚMERO DE CONSUMIDORES SIGNIFICATIVAMENTE AJUSTADOS SITUADOS NAS DUAS PRIMEIRAS DIMENSÕES SIGNIFICATIVAS $(P \leq 0,05)$ DO MDPREF PARA 0 ATRIBUTO TEXTURA ( $\mathrm{N}=117$ CONSUMIDORES)}

\begin{tabular}{lccccc}
\hline Atributos & $\begin{array}{c}\text { Total } \\
\text { ajustado }\end{array}$ & $\begin{array}{c}\text { Superior } \\
\text { direito }\end{array}$ & $\begin{array}{c}\text { Inferior } \\
\text { direito }\end{array}$ & $\begin{array}{c}\text { Superior } \\
\text { esquerdo }\end{array}$ & $\begin{array}{c}\text { Inferior } \\
\text { esquerdo }\end{array}$ \\
\hline Textura & 72 & 30 & 28 & 10 & 4 \\
\hline
\end{tabular}

O MDPREF gerado para a aceitação global (impressão global) dos biscoitos gerou apenas uma dimensão significativa e assim as elipses com 95\% de confiança (Figura 3) não puderam ser calculadas. Porém, clara segmentação dos consumidores pode ser observada em torno das amostras BSUCP e BSCSP situadas nos quadrantes à direita do Mapa e em menor extensão BSTEP, indicando a preferência da maioria dos consumidores (notadamente pelas duas primeiras).
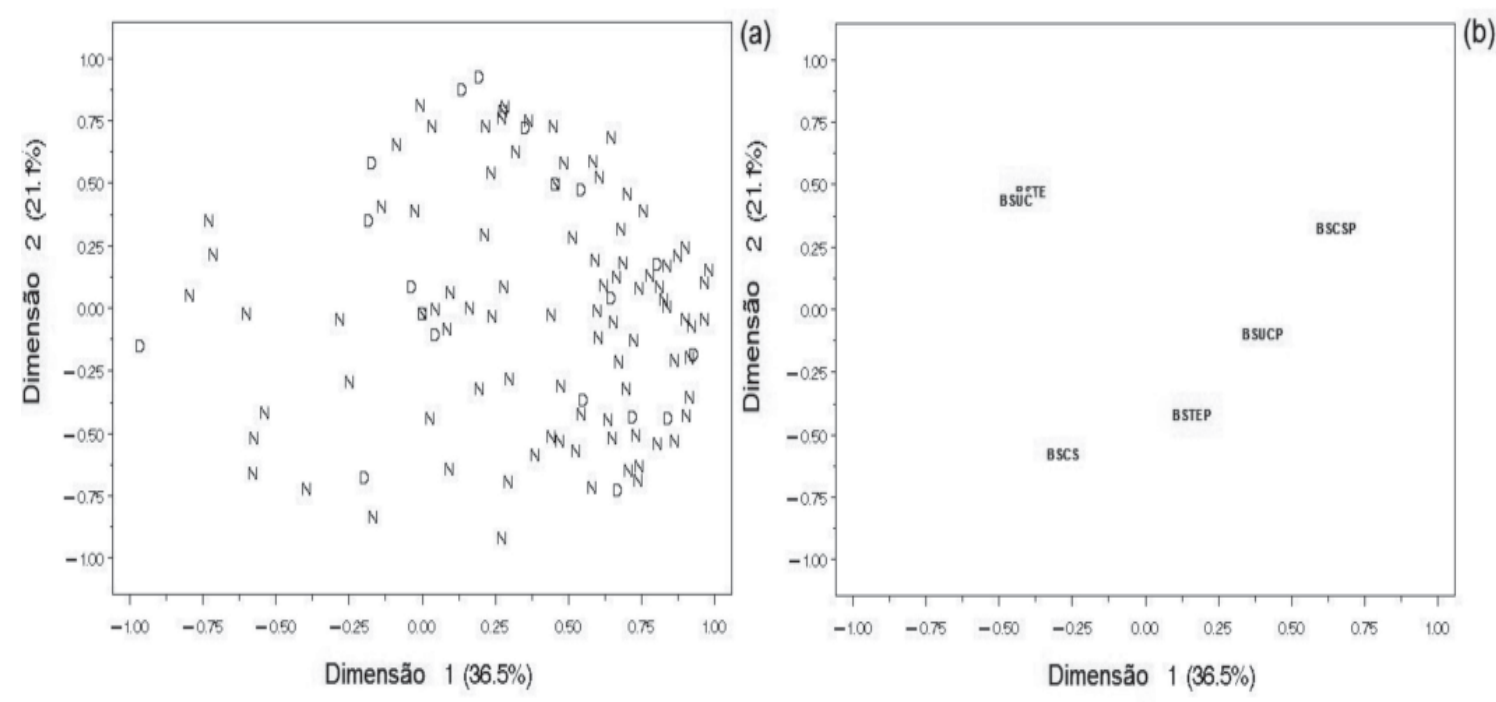

FIGURA 3 - MAPA INTERNO DE PREFERÊNCIA INDICANDO A POSIÇÃO DOS CONSUMIDORES ( $N=117$, DIABÉTICOS “D” E NÃO DIABÉTICOS "N") (A) E DAS AMOSTRAS DE BISCOITO (B) QUANTO À IMPRESSÃO GLOBAL 


\subsection{ANÁLISE DE INTENÇÃO DE COMPRA}

A intenção de compra dos consumidores com relação às formulações desenvolvidas foi avaliada para investigar se os atributos analisados e as médias obtidas de fato influenciaram a decisão da compra dos consumidores estudados.

A Tabela 7 mostra que as formulações BSCSP e BSUCP não diferiram entre si e que obtiveram médias de intenção de compra significativamente superiores $(p \leq 0,05)$ às demais. Os escores obtidos por essas duas formulações foram superiores ao valor 4 (referente ao termo "talvez comprasse, talvez não comprasse") na escala de intenção de compra. Já as notas das demais formulações encontram-se abaixo desse valor, indicando que os consumidores possivelmente não comprariam essas amostras.

A intenção de compra média dos consumidores diabéticos não mostrou diferença entre as amostras com relação a esse quesito, enquadrando-se entre os termos "talvez comprasse/talvez não comprasse" e "certamente compraria". Em contraste, a maioria das amostras obteve médias entre os termos "talvezcomprasse/talveznãocomprasse"e "possivelmentenãocompraria"entreogrupodosnão diabéticos. Assim, observa-se que a percepção dos consumidores diabéticos com relação aos biscoitos dietéticos formulados nesta pesquisa difere da percepção dos indivíduos não portadores da doença.

\section{TABELA 7 - VALORES MÉDIOS DE INTENÇÃO DE COMPRA DE TODOS OS CONSUMIDORES, E DOS SEGMENTOS DOS DIABÉTICOS (PMD) E NÃO DIABÉTICOS (NPD) COM RELAÇÃO AOS BISCOITOS DIETÉTICOS RICOS EM FIBRAS E FORMULADOS COM DIFERENTES EDULCORANTES}

\begin{tabular}{lccc}
\hline \multirow{2}{*}{ Amostras $^{2}$} & \multicolumn{3}{c}{ Intenção de compra $^{1}$} \\
\cline { 2 - 4 } & $\mathbf{P M D}(\mathbf{n}=\mathbf{2 0})$ & $\mathbf{N P D}(\mathbf{n}=\mathbf{9 7})$ & Total $^{(\mathbf{n}=\mathbf{1 1 7})}$ \\
\hline BSUC & $5,60^{\mathrm{a}}$ & $3,27^{\mathrm{b}}$ & $3,67^{\mathrm{b}}$ \\
BSUCP & $6,00^{\mathrm{a}}$ & $4,48^{\mathrm{a}}$ & $4,73^{\mathrm{a}}$ \\
BSCS & $5,35^{\mathrm{a}}$ & $3,44^{\mathrm{b}}$ & $3,77^{\mathrm{b}}$ \\
BSCSP & $6,15^{\mathrm{a}}$ & $4,60^{\mathrm{a}}$ & $4,86^{\mathrm{a}}$ \\
BSTE & $5,45^{\mathrm{a}}$ & $3,09^{\mathrm{b}}$ & $3,50^{\mathrm{b}}$ \\
BSTEP $^{\mathrm{a}}$ & $5,05^{\mathrm{a}}$ & $3,62^{\mathrm{b}}$ & $3,86^{\mathrm{b}}$ \\
\hline DMS $^{4}$ & $1,62^{\mathrm{a}}$ & 0,55 & 0,74 \\
\hline
\end{tabular}

\footnotetext{
${ }^{1}$ Médias com letras em comum na mesma coluna não diferem significativamente entre si a $p \leq 0,05$ pelo teste de Tukey, ${ }^{2} \mathrm{BSUC}=$ biscoito contendo a mistura de edulcorantes sucralose e acessulfame-K, BSUCP = biscoito contendo a mistura de edulcorantes sacarina e ciclamato de sódio e polidextrose; BSCS = biscoito contendo o edulcorante estévia; BSCSP = biscoito contendo a mistura de edulcorantes sucralose e acessulfame-K e polidextrose, BSTE = biscoito contendo a mistura de edulcorantes sacarina e ciclamato de sódio; e BSTEP = biscoito contendo o edulcorante estévia e polidextrose; ${ }^{3} \mathrm{DMS}=$ Diferença mínima significativa.
}

O MDPREF gerado com os dados de intenção de compra dos consumidores encontra-se na Figura 4 (a e b). Juntas, as duas primeiras dimensões explicaram $60,4 \%$ da variação entre as amostras com relação à intenção de compra dos consumidores. As elipses com $95 \%$ de confiança das formulações BSUCP e BSCSP ocuparam regiões muito próximas, indicando notas muito similares. Pode-se dizer o mesmo das formulações BSTE e BSUC.

A concentração dos consumidores nos quadrantes situados a direita da Figura 4, junto das formulações BSUCP e BSCSP, evidenciam que ambas obtiveram a preferência dos consumidores em relação ao quesito intenção de compra. Dos 117 consumidores que realizaram o teste, 54 ficaram alocados fora da elipse com 95\% de confiança, indicando que esses foram os consumidores significativos ajustados pelo modelo utilizado na elaboração do Mapa. Desses, 12 pertenciam ao grupo dos 20 consumidores diabéticos (60\%) e 42 ao grupo dos 97 consumidores não portadores de diabetes $(43 \%)$, sugerindo maior discriminação dos diabéticos na escolha dos biscoitos formulados. 

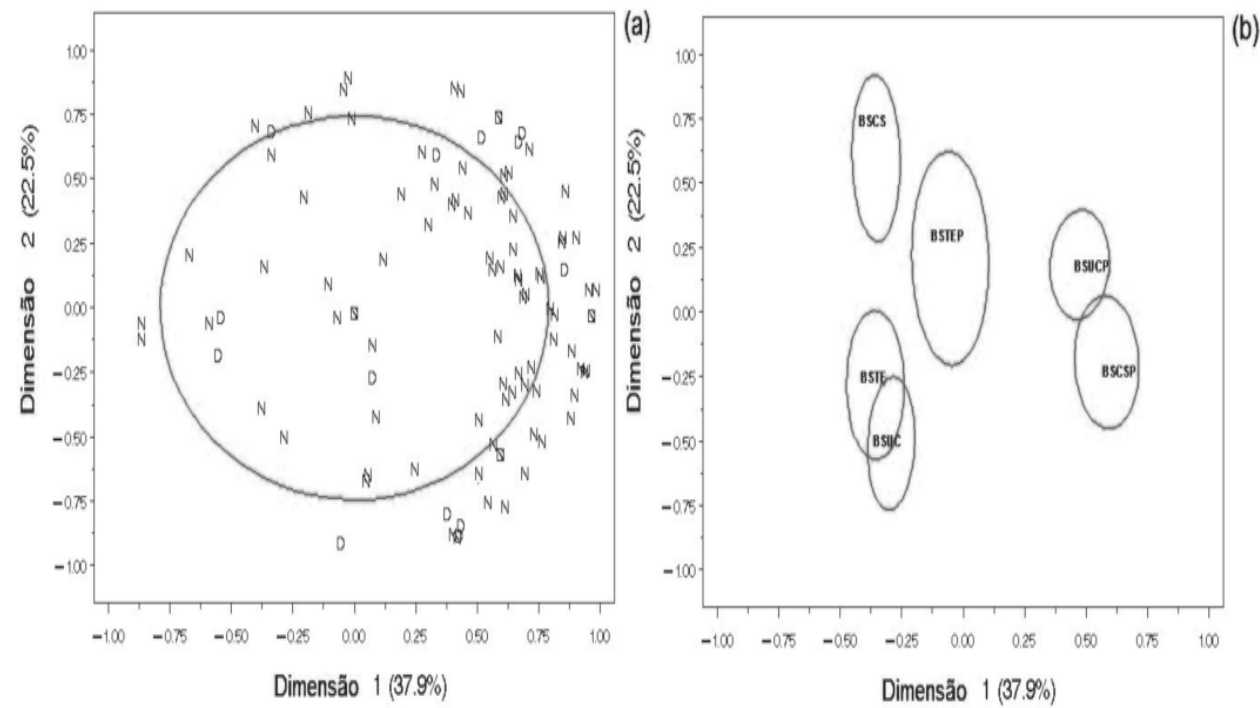

\section{FIGURA 4 - MAPA INTERNO DE PREFERÊNCIA INDICANDO A POSIÇÃO DOS CONSUMIDORES (N=117, DIABÉTICOS “D” E NÃO-DIABÉTICOS “N”) (A) E DAS AMOSTRAS DE BISCOITO (B) QUANTO À INTENÇÃO DE COMPRA}

\section{CONCLUSÃO}

A substituição de açúcar refinado por adoçantes comerciais em duas formulações de biscoitos dietético, ou seja, uma contendo polidextrose e a mistura de sucralose e acessulfame-K e, a outra, polidextrose e a mistura de ciclamato e sacarina sódica não interferiu na aceitabilidade do produto junto a pacientes portadores de diabetes. Os níveis de aceitação dos biscoitos por parte desses consumidores foram maiores do que os dos não portadores da doença. Portanto, o uso de adoçantes em biscoitos elaborados com farinha de casca de maracujá e fécula de mandioca tornase importante opção de produtos dietéticos para alimentação de indivíduos com Diabetes Mellitus, os quais são consumidores habituais desses edulcorantes.

\section{ABSTRACT \\ IMPACT OF SWEETENERS IN DIETETIC BISCUITS ACCEPTANCE FOR DIABETIC AND NON DIABETIC CONSUMERS}

In this study the acceptance of sensory parameters for biscuit formulations with different dietary sweeteners and polydextrose addition were evaluated by consumers with and without Diabetes Mellitus. The results indicated that the use of polydextrose as a substitute for sucrose significantly increased the acceptance of biscuits $(p \leq 0.05)$ from consumers which do not often eat dietetic products. Dietetic biscuits evaluated by consumers with diabetes had significantly higher levels of acceptance $(p \leq 0.05)$ for most attributes. The internal preference mapping suggested higher tendency of consumers used to sweeteners to present differed judgment.

KEY-WORDS: SENSORY ANALYSIS; DIETETIC BISCUIT; SWEETENER.

\section{REFERÊNCIAS}

1 BATTOCHIO, J.R.; CARDOSO, J.M.P.; KIKUCHI, M.; MACCHIONE. M.; MODOLO. J.S.; PAIXAO, A.L.; PINCHELLI, A. M.; SILVA, A.R.; SOUSA, V.C.; WADA, J.K. A.; WADA, J. K. A.; BOLLINI, H. M. A. Perfil sensorial de pão de forma integral. Ciência e Tecnologia de Alimentos, v. 26, n. 2, p. 428-132, 2006.

2 BRASIL. Ministério da Saúde. Agência Nacional de Vigilância Santária. Portaria $n^{\circ} 29$, de 13 de janeiro de 1998. Aprova o regulamento técnico referente a alimentos para fins especiais. Diário Oficial [da] República Federativa do Brasil, Brasília, DF, seção I-E, p. 8, 15 de janeiro de 1998a. 
3 BRASIL. Ministério da Saúde. Agência Nacional de Vigilância Sanitária. Portaria n 27, de 13 de janeiro de 1998. Aprova o regulamento técnico referente à informação nutricional complementar (declarações relacionadas ao conteúdo de nutrientes). Diário Oficial [da] República Federativa do Brasil, Brasília, DF, Seção 1, p. 1244, 16 de janeiro de 1998 b.

4 CAMPOS, M. B. Sucralose e acessulfame-K: a revolução em adoçantes. Food Ingredients, v. 17, p.18-21, 2000.

5 CÂNDIDO, L. M. B.; CAMPOS, A. M. Alimentos para fins especiais: dietéticos. São Paulo: Varela, 1996.432 p.

6 CARDELLO, H. M. A. B.; SILVA, M. A. A. P. da; DAMASIO, M. H. Análise tempo-intensidade dos gostos doce e amargo de extrato de folhas de estévia (Stévia rebaudiana Bertoni) em doçura equivalente a sacarose. Ciência e Tecnologia de Alimentos, v. 19 , n. 1 , p. 163-169, 1999

7 CASTRO, A. G. P. de.; FRANCO, L. J. Caracterização do consumo de adoçantes alternativos e produtos dietéticos por indivíduos diabéticos. Arquivos Brasileiros de Endocrinologia \& Metabologia, v. 46, n. 3, p.280-287, 2002

8 GOMES, C. R.; VISSOTTO, F. Z.; FADINI, A. L; FARIA, E. V.; LUIZ, A. M. Influência de diferentes agentes de corpo nas características reológicas e sensoriais de chocolates diet em sacarose e light em calorias. Ciência e Tecnologia de Alimentos, v. 27, n. 3, p. 787-792, 2007

9 GREENHOFF, K.; MAcFIE, H. J. H. Preference mapping in practice. In: MAcFIE, H. J. H.; THOMSON, D. M. H. (Ed.). Measurement of food preferences. London: Blackie Academic and Professional, 1994. p.137- 166.

10 IOSHII, S. H.; TOLEDO, M. C. F. Avaliação da ingestão diária potencial de edulcorantes em Curitiba. Boletim do CEPPA, v. 12 , n. 2, p. 139-151, 1994.

11 MAcFIE, H. J.; BRATCHELL, N.; GREENHOFF, K.; VALLIS, L. V. Designs to balance the effect of order of presentation and first-order carry-over effects in hall tests. Journal of Sensory Studies, v. 4, n.2, p.129-148, 1989.

12 MALIK, A.; JEYARANI, T.; RAGHAVAN, B. A comparison of artificial sweeteners' stability in a lime-lemon flavored carbonated beverage. Journal of Food Quality, v. 25, n. 1, p.75- 82, 2002

13 MEILGAARD, M.; CIVILLE, G. V.; CARR, B. T. Sensory evaluation techniques. $3^{\text {rd }}$ ed. Boca Raton: CRC, 1999.390 p.

14 MUÑOZ, A. M.; CIVILLE, G. V.; CARR, B. T. Sensory evaluation in quality control. New York: Van Nostrand Reinhold, 1992. $240 \mathrm{p}$.

15 OLIVEIRA, A. P. V.; FRASSON, K.; ALMEIDA, T. C. A.; BENASSI, M. T. Aceitação de sobremesas lácteas dietéticas e formuladas com açúcar: teste afetivo e mapa de preferência interno. Ciência e Tecnologia de Alimentos, v.24, n.4, p.627633, 2004.

16 OLIVEIRA, P. B.; FRANCO, L. J. Consumo de adoçantes e produtos dietéticos por indivíduos com diabetes melito tipo 2 , atendidos pelo Sistema Único de Saúde em Ribeirão Preto, SP. Arquivos Brasileiros de Endocrinologia \& Metabologia, v. 54, n.5, p. $455-462,2010$

17 PINHEIRO, M. V. S.; PENNA, A. L. B. Substitutos de gordura: tipos e aplicações em produtos lácteos. Alimentos e Nutrição, v. 15 , n. 2, p. $175-186,2004$.

18 SAS INSTITUTE. Statistical analysis system. Release 6.08, software. Cary, 2009. 620 p.

19 STATSOFT INC. Statistica (data analysis software system). Version 8.0. Tulsa, 2009. CD-ROM.

20 SANTANA, F. C.; SILVA, J. V.; SANTOS, A. J. A. O.; ALVES, A. R.; WARTHA, E. R. S. A.; MARCELLINI, P. S.; SILVA, M. A. A. P. Desenvolvimento de biscoito rico em fibras elaborado por substituição parcial da farinha de trigo por farinha da casca do maracujá amarelo (Passiflora edulis flavicarpa) e fécula de mandioca (Manihot esculenta crantz). Alimentos e Nutrição, v. 22, n. 3, p. 391-399, 2011.

21 SILVA, M. R.; SILVA, M. S.; MARTINS, K. A.; BORGES, S. Utilização tecnológica dos frutos de jatobá-do-cerrado e de jatobáda-mata na elaboração de biscoitos fontes de fibra alimentar e isentos de açúcares. Ciência e Tecnologia de Alimentos, v. 21 , n. 2, p.176-182, 2001.

22 SOUSA, G. Uso de adoçantes e alimentos dietéticos por pessoas diabéticas. 2006. 64 p. Dissertação (Mestrado em Enfermagem Geral e Especializada), Universidade de São Paulo, Ribeirão Preto, São Paulo, 2006.

23 STONE, H.; SIDEL, J. Sensory evaluation practices. $3^{\text {rd }}$ ed. New York: Academic Press, 2004. 377 p.

24 TOZZETO, A. Controle de qualidade de edulcorantes em adoçantes comerciais via espectrometria e métodos de calibração multivariada. 2005. 144 p. Dissertação (Mestrado em Ciência e Tecnologia de Alimentos), Universidade Estadual de Ponta Grossa, Ponta Grossa, 2005.

25 VILLANUEVA, N. D. M. Avaliação do desempenho de quatro métodos de escalonamento em testes sensoriais de aceitação utilizando modelos normais aditivos de análise da variância e mapas internos de preferência. 2003 . 140 p. Tese (Doutorado em Alimentos e Nutrição), Universidade Estadual de Campinas, Campinas, 2003.

26 VISSOTTO, F. Z. Gorduras especiais: aplicação em recheios. Chocotec Informativo, Campinas, v.3, n.4, p.3, 1997. 